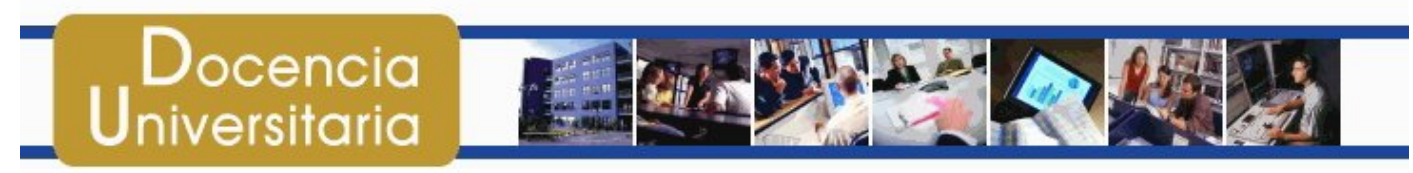

Revista Digital de Investigación en Dacencia Universitaria / Aña 3 - № 2- Dic. 2007

\title{
El desarrollo del personal académico de las universidades venezolanas: propuesta para diseñar e instrumentar un programa de formación
}

\author{
Faculty improvement in Venezuelan universities: \\ A proposal for the design and implementation of a development program
}

\author{
Migdy Chacín \\ Mariangelina de Kolster \\ Zobeida Ramos
}

\section{Resumen}

Las universidades venezolanas se desenvuelven en un mundo globalizado y cambiante al cual le intentan dar respuesta instrumentando acciones relacionadas al mejoramiento de los profesores. Para acometer esa tarea, se propone un modelo de formación profesoral fundamentado en la satisfacción de necesidades individuales, institucionales y contextuales. Se describen las etapas de diseño e instrumentación del programa de formación, concluyendo con una reflexión sobre la importancia de atender apropiadamente lo relativo al desarrollo de los profesores universitarios $\mathrm{y}$ al compromiso que los gerentes educativos deben adquirir con el uso eficiente de los recursos y la gestión de instituciones que respondan cabalmente a lo que la sociedad requiere.

Palabras clave: desarrollo del talento humano, desarrollo profesoral en las universidades venezolanas, modelos de programas de formación, etapas y estrategias para el diseño e instrumentación de un programa de desarrollo para el profesor universitario

\begin{abstract}
Venezuelan universities develop in a changing and globalized word. They must fulfill what has been established in governmental policies regarding faculty development. This paper proposes a model for faculty development based on individual and societal needs.Design and implementation stages of the program are described in detail and followed by discussion of the importance of enough government support, university leaders' commitment to efficient administration of funds and the implementation of programs that produced the changes needed by society.
\end{abstract}

Key words: human development, faculty development in Venezuelan universities, development programs models, stages and strategies in development programs. 


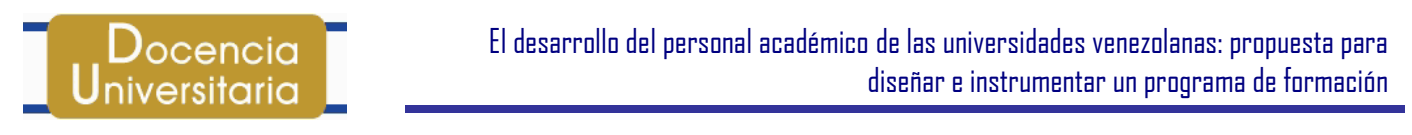

Аก̃̃ 3- N²- Dic. 2007

\section{Introducción}

El interés por el talento humano es compartido por los gestores organizacionales y los estudiosos de la administración, quienes, sobre la base de teorías, experiencias e investigaciones, buscan formas de potenciar a los trabajadores de una organización para un mejor accionar personal y profesional.

Las universidades venezolanas, como organizaciones, comparten esa necesidad. Diversos son los elementos en los que se patentiza el interés por planificar su gestión y evaluar el cumplimiento de las metas. Sin embargo, estos planes no parecen ahondar lo suficiente en el desarrollo del personal académico. Si bien en los planes gubernamentales e institucionales se formulan metas respecto al otorgamiento de recursos para la investigación y el desarrollo del profesorado, no siempre se programan esas acciones ni se consideran todos los casos como parte de las prioridades organizacionales. Esta situación puede provocar que los esfuerzos invertidos sean poco productivos y pertinentes respecto a lo que las organizaciones y la sociedad requieren.

En las instituciones venezolanas de educación superior, los procesos relativos a la formación y desarrollo de los académicos se encuentran regidos por normativas legales generales, que reglamentan la selección, ingreso, ubicación en el escalafón, permanencia y ascenso de los profesores. Además, cada institución establece, dentro de este marco, derechos y obligaciones adecuados a sus particulares características y circunstancias para reglamentar todo lo referido a la carrera docente. En la ley general, sin embargo, no se especifica una normativa directamente relacionada con el desarrollo del personal académico; solamente en las reglamentaciones internas de las universidades de muy reciente creación se incluyen artículos referidos a este tema, estableciendo fundamentalmente los requisitos a cumplir en la primera etapa de la carrera académica. Una de las características del sistema de ascenso en Venezuela característica que no es ajena a instituciones docentes en otras partes del mundo - es que la cualidad profesional está ligada a una promoción ya definida en baremos unida a su vez a una correspondiente mejora monetaria. Esto significa que, en principio, la motivación para el desarrollo personal y profesional de los profesores universitarios es eminentemente intrínseca.

Sin embargo, a la luz de los movimientos de calidad en cuanto al desarrollo del talento humano, ese no parece ser el camino ideal, ya que las decisiones respecto a la formación profesional no deben responder exclusivamente a las necesidades individuales. Es sabido que cuando una organización posee objetivos bien determinados, puede alentar a sus integrantes a alcanzar metas de desarrollo profesional; es decir, si el desarrollo 


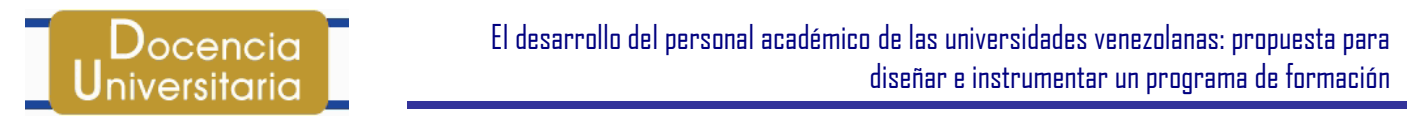

Аก̃̃ 3- N²- Dic. 2007

profesional se realizara en las universidades como fruto de una convergencia entre las aspiraciones personales y las de la organización -expuestas en políticas claras -, se alcanzaría un doble propósito: se evitaría, por una parte, que se dispersaran los esfuerzos de los integrantes de la organización y, además, se haría más explícita la difusión de las oportunidades de desarrollo, adecuándolas al interés de la propia institución y asegurándose que sean compatibles con sus políticas y planes.

Esta es la realidad que sirve de contexto para el presente trabajo, en el que se formula una propuesta para diseñar e instrumentar programas concernientes al desarrollo del personal académico de las universidades. La propuesta es producto de una extensa indagación sobre lo que proponen experimentados autores respecto a la administración del talento humano, de lo que se practica en las universidades venezolanas y de la experiencia de las autoras sobre gestión para el desarrollo del profesor universitario. Asimismo, se nutre de las sugerencias de numerosos profesores universitarios sobre lo que debería caracterizar al quehacer institucional respecto a la instrumentación de programas de desarrollo de la carrera docente a nivel superior.

\section{El problema}

Hablar sobre la importancia del desarrollo del talento humano resulta casi innecesario hoy en día, ya que existe consenso sobre la velocidad a la que avanza el conocimiento y la necesidad de seguir ese ritmo. En el caso del profesor universitario, la importancia de su desarrollo profesional es aún más imperativa, dado el compromiso que la propia profesión reclama: la formación de una generación de relevo y de una actitud proactiva hacia el aprendizaje independiente y continuo.

Las universidades, lógicamente, tienen que dedicar gran parte de sus esfuerzos a la materialización de los conceptos de actualización y aprendizaje permanente, explicitados ambos en las leyes respectivas. De allí que en el subsistema de educación superior venezolano se establezca un desideratum hacia las acciones que propenden al desarrollo del profesor, respaldados por leyes, reglamentos y estructuras organizacionales. Pero las acciones concernientes a ello se instrumentan de forma independiente (como resultado de la iniciativa personal) o conjunta (con la anuencia de los decanatos), responden a las necesidades expresadas por los profesores y, ocasionalmente, se insertan al interior del marco de los requerimientos institucionales y sociales. Pese a esto, la gerencia de las universidades no parece interesada en enmarcar sus proyectos de desarrollo de acuerdo a su visión, su misión y sus objetivos institucionales, siendo también pocas las ocasiones en las que se definen políticas para el perfeccionamiento docente. 


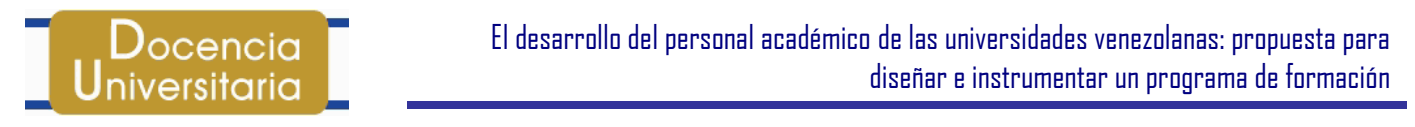

Аก̃̃ 3- N²- Dic. 2007

Por ello, el casi siempre exiguo presupuesto asignado por los organismos públicos competentes en la materia (en el caso de las universidades del Estado), o el que la institución destina (en el caso de las universidades privadas), no atiende, en todos los casos, las solicitudes de los docentes de forma equitativa. Tampoco se verifica que el esfuerzo se encuentre en consonancia con las necesidades organizacionales (existentes, aunque no se hayan expresado), lo cual puede provocar que se ejecute la asignación monetaria de forma descontextualizada. Por estas razones, los recursos invertidos en la formación del personal académico no resultan en beneficios tangibles ni para la institución ni para la sociedad.

Teniendo en cuenta la realidad anteriormente descrita, y con la finalidad de mejorar la situación actual, se formula en el presente trabajo una propuesta para diseñar e instrumentar un programa para el desarrollo del profesor universitario. La propuesta se sustenta en las siguientes hipótesis:

- Toda institución universitaria debe definir su misión, visión y objetivos para determinar sus niveles de calidad, eficiencia y eficacia, dadas las necesidades del contexto social y de su contexto particular.

- Las actividades de formación y desarrollo son necesarias para la actualización del profesor de educación superior, así como para el mejoramiento de los niveles de desempeño laboral.

- Las instituciones universitarias deben tener un compromiso mayor con la formación, actualización y desarrollo de su personal académico, atendiendo al compromiso adquirido por el profesor respecto a la creación de conocimientos y la formación de una generación de relevo.

- Cada institución debe generar su propio plan de desarrollo, debiendo ser este definido de acuerdo a las características y políticas institucionales, e instrumentado a partir un modelo que tome en consideración tanto las necesidades del profesor como las de la institución y las de la sociedad.

\section{Marco teórico referencial}

Al estudiar la relevancia del desarrollo del talento humano, (Andrade, 2003) señala que la organización requiere que los individuos desarrollen su carrera y adquieran un compromiso con el progreso de la institución, pues de ello depende el suyo. De allí radica la importancia de esmerarse tanto en los procesos de reclutamiento y selección de personal, así como en los de evaluación del desempeño, el uso de sistemas de compensación, y la realización de actividades de formación y desarrollo profesional. 


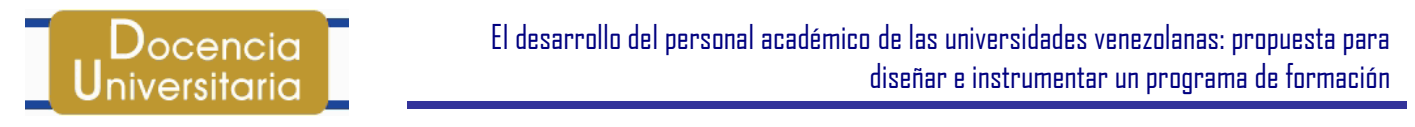

Аก்̃ 3- N²- Dic. 2007

Estos elementos, en gran medida, garantizarán el mejoramiento de los niveles de calidad organizacional.

En cuanto a la formación señala Cerna ${ }^{1}$ que se trata de un proceso sistemático que busca la modificación del comportamiento, la adquisición de conocimientos y el aumento de la motivación en los empleados, con el fin de mejorar la relación entre las características del trabajador y las de la organización. De acuerdo a este orden de ideas, debe considerarse la formación como inversión estratégica y componente básico para la competitividad. En el caso específico de los profesores universitarios Castillo y Cabrerizo $^{2}$ señalan que el concepto de formación se encuentra referido a planeamientos formales de procesos didácticos que posibilitan, desde el inicio de su carrera, la capacitación, preparación o perfeccionamiento del docente para su desarrollo y mejora profesional.

Si intentáramos caracterizar el contexto en el cual se desenvolverán las universidades latinoamericanas en el presente siglo, algunas de sus realidades serían las siguientes: funcionarían en un clima de autonomía bastante consolidada, habría dado sus frutos la democratización como principio, se habrían resuelto los requerimientos de la masificación de la enseñanza debido al aumento de instituciones educativas, y se encontrarían con mayor regularidad equipos de investigadores multidisciplinarios, los cuales -aunque dispersos en la geografía mundial - trabajarían en común hacia el logro de metas similares. Sin embargo, algunos de los problemas que actualmente enfrentan las universidades latinoamericanas muy probablemente se mantendrían constantes en un futuro. Entre ellos, podrían encontrarse los siguientes: la existencia de severas limitaciones financieras; la falta de articulación entre los distintos niveles educativos; la problemática derivada de la necesidad de actualización permanente del currículum; y, como consecuencia de lo anterior, la necesidad de mejorar permanentemente la calidad académica del profesorado.

Son numerosos los documentos en los que se que evidencia la preocupación por elevar los niveles de calidad del profesorado de las universidades venezolanas, debido a la necesidad de adecuación que tendrán dichas instituciones para acometer un cambio sustantivo en su quehacer cotidiano. En este sentido, el Ministerio de Educación, Cultura y Deportes de la República Bolivariana de Venezuela señala en el libro sobre Políticas y Estrategias Para el Desarrollo de la Educación Superior 2000-2006³ , que una de las metas es elevar la calidad académica de las instituciones y mejorar su

\footnotetext{
${ }^{1}$ Cerna 2004

${ }^{2}$ Castillo y Cabrerizo 2005

${ }^{3}$ Ministerio de Educación, Cultura y Deportes de la República Bolivariana de Venezuela 2001
} 


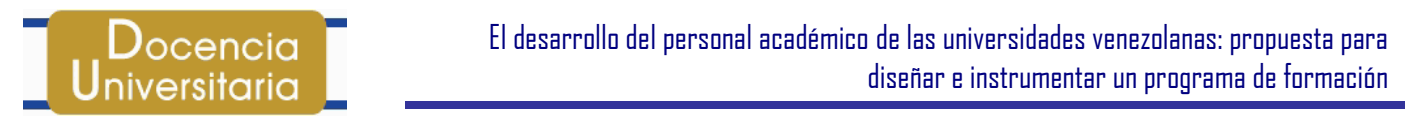

Аก̃̃ 3- N²- Dic. 2007

eficiencia institucional. Entre las estrategias dirigidas específicamente a asistir al profesor universitario en su proceso de desarrollo se señalan las siguientes:

- El diseño e implantación de un sistema de evaluación y acreditación institucional para atender las peculiaridades de cada institución y como medio para contribuir con las transformaciones requeridas en términos de calidad académica y de gestión institucional.

- El diseño e implantación de un sistema nacional de carrera académica que premie el esfuerzo personal como compensación al desempeño académico.

- La elaboración y desarrollo de planes integrales de formación del profesorado que incorporen las dimensiones sociales, políticas, éticas y pedagógicas implicadas en el quehacer educativo.

Asimismo, expertos en el tema destacan la importancia de incrementar los niveles de calidad de las universidades venezolanas a partir de acciones para el desarrollo del personal académico. Villarroel ${ }^{4}$ indica, por ejemplo, que la crisis actual de la educación superior venezolana es relativo a la calidad, concepto que, en su opinión, debe definirse y operacionalizarse dentro del contexto de cada universidad. Sostiene, además, que la calidad universitaria se encuentra dada por la "sumatoria de las calidades de sus elementos” (Villarroel 2007: 45), entre los cuales se incluye la cualificación y formación del profesorado.

En relación al concepto de productividad, que se equipara en algunos casos al de calidad de las instituciones educativas, Cortés y González indican que “a mayor capacitación y actualización, mayor será la productividad en términos de innovación, creatividad y participación” (Cortés y González 2006: 137). De acuerdo a ello, Sánchez ${ }^{5}$ apunta que los programas de desarrollo profesional deben: a) estar dirigidos a profesores en pleno ejercicio; b) tener como finalidad la formación pedagógica inicial y permanente; y c) concebirse como un proceso planificado de crecimiento y mejora del conocimiento en el área profesional, de generación de actitudes positivas hacia el trabajo y la institución, y de satisfacción de las necesidades personales, institucionales y sociales. Asimismo, Medina (citado por Sánchez) ${ }^{6}$, indica que el desarrollo profesional es la construcción de la identidad profesional, y que los cambios que este produzca deberán orientarse a los ámbitos pedagógicos, profesionales y personales-sociales. En suma, el objetivo final de un programa de desarrollo para el profesor universitario debe ser la mejora de la calidad docente respecto a la actualización profesional, docencia, investigación, gestión

\footnotetext{
${ }^{4}$ Villarroel 2007

${ }^{5}$ Sánchez 2007

${ }^{6}$ Sánchez 2007
} 


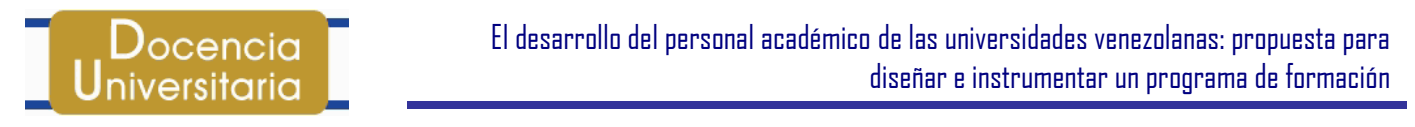

Аก̃̃ 3- N²- Dic. 2007

y extensión como sus funciones primordiales. El programa, por lo tanto, debe dar respuesta a las necesidades individuales y a las de la organización ${ }^{7}$, debiendo ser su eje la preparación que los docentes requieren para asumir los nuevos desafíos científicos de su área específica de conocimiento, así como para responder adecuadamente a las necesidades de sus estudiantes en los distintos contextos.

Para la propuesta del presente trabajo, resulta relevante la consideración planteada por Sánchez ${ }^{8}$ respecto a que la formación surja de la iniciativa del profesorado, contando con la previa aceptación del programa por parte de los docentes. Esto puede lograrse a través de un proceso de sensibilización y participación en el diseño del mismo. El autor destaca también la importancia que tiene el que las acciones del programa reflejen lo expresado en los planes institucionales para la mejora de la oferta académica, tratando también de incorporar en los mismos las necesidades provenientes del contexto institucional.

Autores experimentados en el estudio de contextos de tipo empresarial, como Simon Dolan, Ramón Valle, Susan E. Jackson, Randall Schuler, comentan que "muchas empresas han llegado a la conclusión que ayudar a sus empleados a tomar decisiones referentes a su carrera llevará, en última instancia, a una mayor satisfacción y lealtad hacia la organización. Por lo tanto, se elaboran políticas y se establecen trayectorias y procedimientos de desarrollo de la carrera profesional para ayudar a los empleados a alcanzar sus objetivos a través de programas de gestión de la carrera” (Dolan 2007:206). Esto resulta más importante en el caso de los profesores universitarios, dada su vocación de transmitir y crear conocimiento y valores a las nuevas generaciones.

Los aspectos anteriormente discutidos nos permiten plantear unas hipótesis sobre la propuesta para el diseño y la instrumentación de un programa de desarrollo para el profesor universitario:

- Es necesario formular programas para el desarrollo académico del profesor universitario respaldados con la definición de políticas institucionales.

- La concepción de los programas debe partir de la consideración de las necesidades personales, institucionales y sociales.

- Estos programas deben tener los siguientes componentes: iniciación y formación permanente para la docencia, investigación, extensión y

\footnotetext{
${ }^{7}$ Sánchez 2007

${ }^{8}$ Sánchez 2007
} 


Docencia - El desarrolla del persanal académica de las universidades venezolanas: propuesta para
diseñar e instrumentar un programa de formación

Aก̃̃ 3- № - Dic. 2007

gestión, así como también para la actualización en el área profesional. 


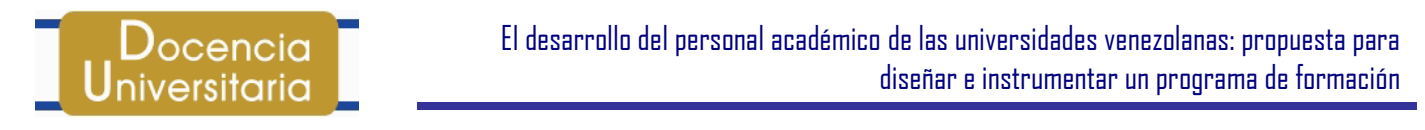

Añ̃ 3- N²- Dic. 2007

\section{El programa de desarrollo: modelos subyacentes para el diseño}

Los programas para la formación y el desarrollo del docente universitario pueden diseñarse de acuerdo a distintos modelos. Peña ${ }^{9}$ hace referencia a cinco de ellos. Los cuatro primeros se encuentran basados en los siguientes procesos: 1) perfeccionamiento; 2) evaluación; 3) indagación; 4) organización; y 5) modelo integrador.

El primer modelo (de perfeccionamiento) brinda respuesta a las necesidades personales del docente y exige a las instituciones estar abiertas y dispuestas a satisfacer los requerimientos individuales, ofreciendo diferentes alternativas que propicien el desarrollo de sus académicos. El segundo modelo (de evaluación) tiene como propósito mejorar el ejercicio profesional, dados los resultados de la evaluación institucional y la evaluación del desempeño del docente, realizados ambos sobre la base de un ideal preconcebido.

El tercer modelo (de indagación) se sustenta en la premisa de que el individuo busca las fuentes de su perfeccionamiento a través de la observación y el uso de estrategias para la resolución de problemas.

El cuarto modelo (organizacional) se centra en la satisfacción de las necesidades institucionales, la mejora de las condiciones de trabajo y el cumplimiento de objetivos y metas organizacionales; todo esto debe producir cambios estructurales previamente definidos en armonía con las necesidades de la sociedad.

El quinto modelo que refiere Peña es un Modelo Integrador, al cual adhieren las autoras y que se describirá a continuación.

\section{Propuesta para el diseño del programa}

El Modelo Integrador, elaborado por Peña, surge para superar las limitaciones de los cuatro modelos anteriormente descritos por él. Se fundamenta en tres directrices y concibe el desarrollo profesoral de la siguiente manera:

a) Centrado en la práctica profesional.

b) Sustentado por las necesidades individuales e institucionales.

c) Enriquecido por las actitudes y conductas cooperativas de los profesores.

\footnotetext{
${ }^{9}$ Peña 2003
} 


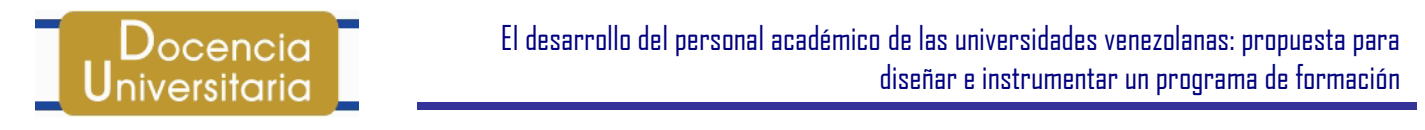

Аก̃̃ 3- N²- Dic. 2007

Las autoras se identifican con el Modelo Integrador, ya que coinciden con Peña en los elementos que deben sustentar la formulación de un programa para el desarrollo del profesor universitario. Asimismo, añaden algunas ideas que incluyen tanto aspectos relevantes aportados por otros modelos de desarrollo, así como elementos considerados importantes por las autoras del presente trabajo:

1. Las necesidades de desarrollo se deben identificar tomando en consideración los resultados obtenidos de la revisión de la misión, visión y objetivos institucionales, de la evaluación del contexto organizacional y de las necesidades planteadas por los miembros de la academia.

2. Se requiere un proceso de evaluación continua del programa, que permita la oportuna corrección de errores y ratificación de aciertos.

3. Para el éxito del programa de desarrollo resulta necesario que las autoridades universitarias propicien un clima de participación desde el diseño del mismo. También es indispensable que todos los involucrados en el programa (la sociedad, la institución y los participantes) adquieran un compromiso con el perfeccionamiento como actividad permanente.

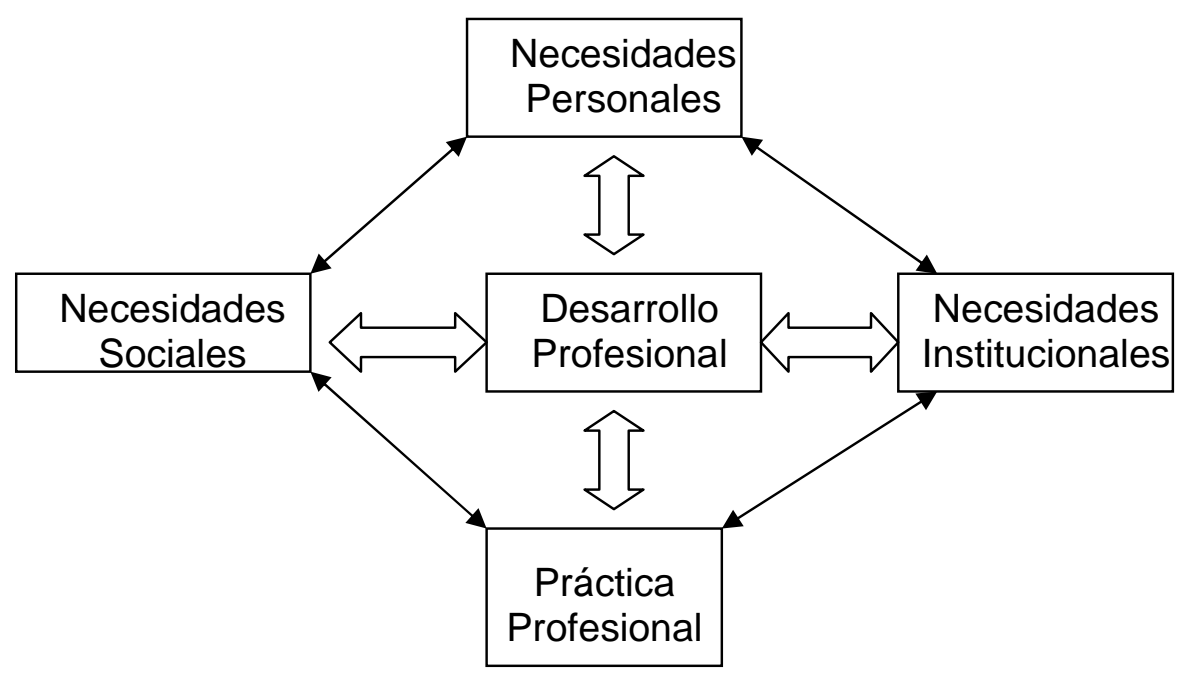

Figura 1 Modelo Integrador propuesto para fundamentar un programa de desarrollo para el profesor universitario.

Fuente: las autoras

La Figura 1 ilustra la integración que debe existir entre cada uno de los elementos que conforman el modelo, los cuales son la fuente (u origen o causa) y consecuencia (o efecto o producto) del otro, por la permanente interacción entre cada uno de ellos. En esta se patentiza la relación sinérgica entre: a) las necesidades personales (expresadas por el individuo de forma particular o con la anuencia de su conjunto de pares); b) 


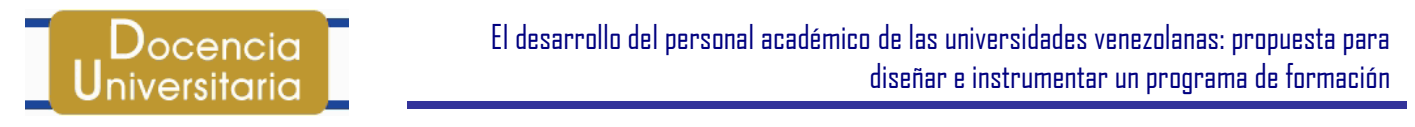

Аก̃̃ 3- N²- Dic. 2007

institucionales (definidas en la misión, visión y objetivos de la organización); c) sociales (definidas por las instancias de planificación nacional o por los requerimientos del contexto); y d) la práctica profesional del profesor universitario (docencia, investigación, extensión y gestión). La conjunción de estos elementos permite el desarrollo profesional, manifestado en distintos niveles, y plasmado en el programa de desarrollo, que debe ajustarse cabalmente a las necesidades del individuo y las de su contexto laboral y social. Las flechas de doble vía expuestas en el modelo demuestran la necesidad de una evaluación permanente e integradora de cada uno de los elementos del mismo en cada una de las fases del programa. Finalmente, el cuadro central de la figura (desarrollo profesional) surge como resultado de la definición de las necesidades. Este proceso debe ser realizado con una amplia participación del usuario del programa y de los miembros de la comunidad en la cual se desenvuelve.

Esto demuestra la importancia de la participación activa de todos los interesados en el diseño del programa de desarrollo profesional: la sociedad, que requiere la formación de profesionales capacitados para satisfacer apropiadamente las necesidades del contexto; la institución de educación superior, enmarcada en la exigencia de leyes, reglamentos y planes operativos; y los profesores universitarios dispuestos a invertir esfuerzo en su formación y actualización profesional.

\section{El diseño del programa}

El proceso de diseño del programa está concebido en etapas. La primera, llamada de diseño o formulación, es un proceso multifacético, en el cual, sobre la base de la integración de las necesidades sociales, institucionales y personales, y utilizando estrategias para motivar la participación, se identifican las características del programa. Esta fase requiere de un continuo monitoreo que implicará la recolección y evaluación de los resultados obtenidos en términos de la productividad de los recursos invertidos (tiempo, dinero, talento humano). Esta etapa está integrada, a su vez por tres sub-etapas que se diferencian entre sí de acuerdo a la meta que buscan alcanzar: conceptualización, definición y divulgación.

5.1 En la sub-etapa de conceptualización se definen las características generales del programa, seleccionando a los responsables de su coordinación y formulación.

Revisando la bibliografía sobre el tema, es importante considerar los siguientes aspectos al planificar un programa de desarrollo para el profesor universitario:

- La definición del ámbito del programa depende del tipo de institución y la misión de ésta, su marco de referencia debe integrar las necesidades sociales, 


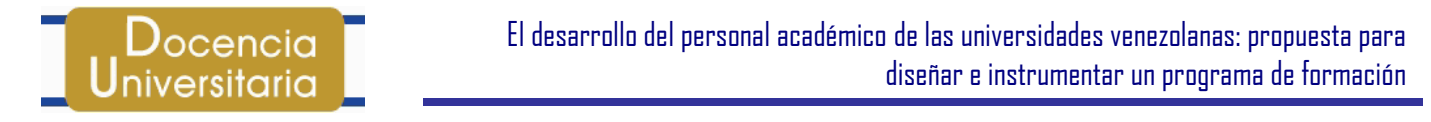

Аก̃̃ 3- N²- Dic. 2007

institucionales e individuales, considerando las características de la población que se asistirá y su motivación para aprender.

- El plan institucional debe ser realista con las metas. No debe ser demasiado ambicioso, especialmente en su primera etapa de ejecución. Asimismo, resulta necesario que la organización desee y se encuentre totalmente dispuesta a apoyar de distintas formas de aprendizaje. Lo mismo debe ocurrir con quienes quieran aprender.

- Un programa de desarrollo debe ser: a) atractivo, motivando al profesor a involucrarse en el mismo y a invertir los esfuerzos que sean necesarios para lograr sus metas de profesionalización; b) competitivo, generando una preocupación institucional y personal sobre la calidad que incite al profesor a destacarse entre sus pares y que, asimismo, lo recompense (no solo en términos financieros, sino en el reconocimiento a sus méritos); c) diseñado para recompensar al profesor universitario por sus altos niveles de enseñanza en términos de productividad y calidad; y d) permanente, estimulando al docente más allá de los logros diarios, y motivándolo hacia un plan integral de perfeccionamiento casi indefinido.

- Los profesores responderán con entusiasmo a un programa de desarrollo que cuente con las siguientes características: a) recompensa la calidad del desempeño; b) es novedoso en las actividades y estrategias de enseñanza, los contenidos programáticos a estudiar y las competencias a desarrollar; y c) es asequible para la inserción del profesor al mismo.

- El participante del programa es un adulto y, por lo tanto, el programa debe responder y adaptarse a las características, necesidades y particularidades de este tipo de estudiante.

Azócar ${ }^{10}$ et al. señalan que para lograr la participación de la comunidad académica en la planificación del programa pueden utilizarse las siguientes estrategias:

a) Planificación de arriba hacia abajo, en la que intervienen los expertos, que, sobre la base de necesidades institucionales prioritarias previamente establecidas, definen un calendario de eventos y seleccionan a las personas que participarán en los procesos de diseño, instrumentación y evaluación del programa.

b) Planificación de abajo hacia arriba, la cual se nutre de las consultas realizadas a todos los estamentos de la comunidad. Sobre esta base, se delimitan las prioridades y acciones a ejecutar.

\footnotetext{
${ }^{10}$ Azócar 1995
} 


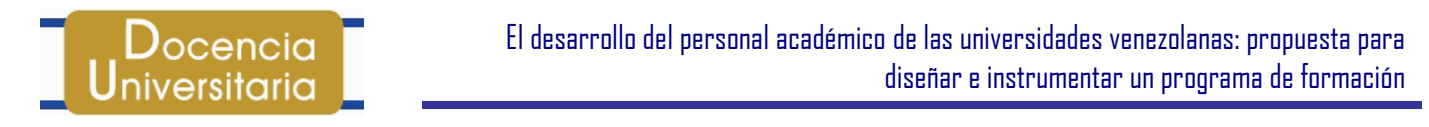

Аก̃̃ 3- N²- Dic. 2007

c) Planificación mediante una combinación de ambas modalidades, en la que se consulta a la gerencia media y a los representantes profesorales sobre el establecimiento del programa. En esta, los Decanos y Jefes de Departamento colaboran en la identificación de las metas a lograr basándose en los conocimientos y destrezas que se desarrollaran de acuerdo a los objetivos institucionales y departamentales.

Otro estrategia sugerida en la bibliografía para conceptualizar el programa es la asesoría de un especialista, usualmente externo a la institución, quien con una limitada participación en la comunidad académica, propone los resultados del programa, reconociendo las estrategias necesarias para la divulgación de las competencias que se aspiran desarrollar.

5.2 La sub-etapa de definición y planificación operativa del programa tiene como propósito distinguir: a) las metas a corto, mediano y largo plazo; b) las actividades a desarrollar; c) las estrategias para instrumentar el programa; y d) los recursos a utilizar (financieros, materiales y humanos). Para ello se formulan las siguientes recomendaciones:

5.2.1 Los programas de desarrollo para el profesor universitario deben contribuir a la formación integral del mismo, mediante la instrumentación de un plan de carrera que atienda tanto las necesidades de crecimiento profesional del individuo, como aquellas vinculadas con su propio desempeño en la institución. El plan de carrera debe incluir los siguientes componentes: inducción, capacitación, perfeccionamiento y actualización profesional. La inducción tiene como objetivo facilitarle al profesor la familiarización e integración al sistema educativo. La capacitación busca la adquisición de destrezas específicas relacionadas con las tareas a desempeñar. Por último, el perfeccionamiento y la actualización ayudan al profesor a mantenerse al día respecto a los avances suscitados en su área profesional.

5.2.2 Las áreas del conocimiento que deben abarcar los programas de desarrollo y perfeccionamiento para el profesor universitario son las siguientes:

- Desarrollo pedagógico, que crea destrezas en la enseñanza, investigación, diseño de instrucción y extensión.

- Desarrollo profesional, que atiende a la necesidad de actualización en el área específica del conocimiento.

- Desarrollo gerencial, que se orienta a formar al profesor para el desempeño de cargos que revistan tareas distintas a la labor docente. Por 


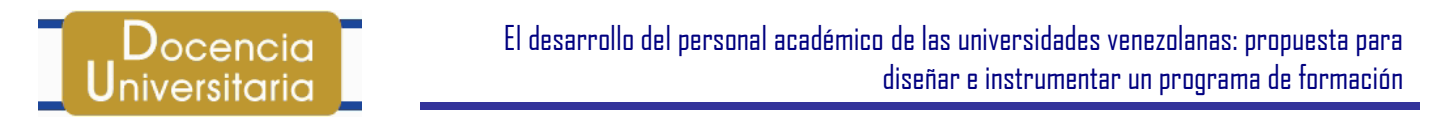

Аก̃̃ 3- N²- Dic. 2007

ejemplo, la supervisión de personal o la administración de recursos financieros.

- $\quad$ Desarrollo personal, que incide en el crecimiento individual, incluyendo el desarrollo de habilidades para la comunicación interpersonal.

En cada área de formación resulta necesario identificar los objetivos de aprendizaje a lograr, los estándares de ejecución, las actividades que se realizarán, las conductas que se desarrollarán y los criterios con los que se evaluarán los resultados de aprendizaje. Esto último puede lograrse también con la participación de expertos que, por una parte, contribuyan a determinar los objetivos de aprendizaje, contenidos y estrategias de enseñanza para cada curso, y que, por la otra, ayuden a la selección o desarrollo de los materiales de instrucción que se utilizarán.

En cuanto a las estrategias de enseñanza, se recomienda aquellas que favorezcan el aprendizaje colaborativo y que incorporen sistemas novedosos de instrucción que el profesor pueda dominar e incorporar a su práctica diaria (Cravener, 2000).

5. 3 La sub-etapa de divulgación tiene como propósito que el programa sea conocido en niveles extra e interinstitucionales, informando especialmente a los sectores que se aspira asistir respecto a la oferta académica, las opciones acordes a las necesidades e intereses personales, y los cambios que pudieran ocurrir al interior del programa. Es muy importante considerar en esta etapa la forma en que se estimulará y promoverá la incorporación y participación de los profesores en la planificación de las diversas actividades de formación y desarrollo, a fin de garantizar el éxito de las mismas (Lawler y King, 1999; Chacín y Figueroa, 1997).

\section{ESTRATEGIAS PARA LA INSTRUMENTACIÓN DEL PROGRAMA.}

Paralelamente a la formulación del programa, deben identificarse las estrategias que permitirán garantizar que el mismo se desarrolle de acuerdo a lo previsto en el plan institucional. Para ello, se sugiere describir con la mayor precisión posible las características del programa que se desea instrumentar, integrándolo, junto a las actividades que la institución pueda realizar, a estrategias de cooperación interinstitucional. Esto permitirá una utilización más racional de los recursos disponibles en organizaciones con similares intereses. Entre ellas, mencionamos las siguientes:

6.1 A nivel interinstitucional es importante el establecimiento de convenios centrados en el perfeccionamiento docente. En estos, cada institución afiliada selecciona un representante y se compromete y aporta un porcentaje de los recursos de los que 


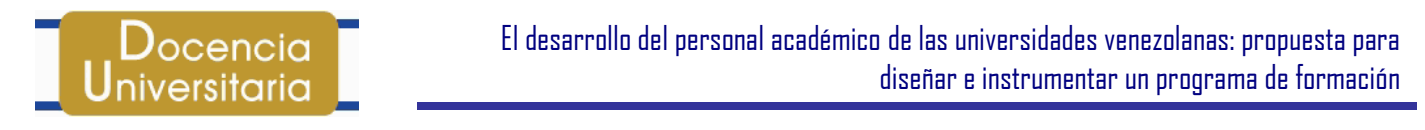

Аก̃̃ 3- N²- Dic. 2007

dispone para el desarrollo del programa. Entre las tareas que deberían realizarse, estarían las siguientes:

- Constituir una red para la administración de programas de perfeccionamiento docente y de oferta de cursos, foros, talleres, jornadas, premiaciones, y becas, entre otros.

- Utilizar las redes de comunicación para el aprendizaje con apoyo del computador, lo que permitiría la coincidencia espacio-temporal de todos aquellos que desearan comunicarse con propósitos comunes.

- Compartir a través de redes de información las experiencias realizadas en las áreas de educación y de investigación, así como el manejo de los recursos humanos, el apoyo institucional y las tecnologías. Esto enriquecería los programas de perfeccionamiento docente en cada una de las instituciones participantes (UNELLEZ, 2003).

- Atender las necesidades propias del programa en cada una de las instituciones que integran la red (UNELLEZ, 2003).

- Contribuir a la constitución de equipos interdisciplinarios de investigación. Esto promovería la producción del conocimiento en el área de perfeccionamiento docente (UNELLEZ, 2003).

- $\quad$ Propiciar el intercambio de recursos para la administración del programa (instalaciones, maquinarias, equipos), así como el intercambio de profesores altamente calificados en funciones de docencia y/o investigación.

6.2 A nivel institucional es imprescindible la creación de una instancia operativa que coordine las labores de diseño, instrumentación, seguimiento y evaluación del programa de desarrollo. Entre sus funciones se encuentran las siguientes:

- Definir conjuntamente con la gerencia alta, la gerencia media y el cuerpo profesoral las políticas y lineamientos generales de desarrollo, evaluación y promoción del docente universitario.

- $\quad$ Proponer la elección de un líder o administrador del programa por parte de la instancia superior.

- Coordinar y asesorar los programas de formación y desarrollo del profesor universitario de cada unidad académica.

- $\quad$ Propiciar la creación de equipos de investigación que identifiquen las variables que inciden en la calidad de la enseñanza universitaria.

- Incluir actividades que motiven a los profesores a involucrarse en el programa de desarrollo. Por ejemplo, el otorgamiento de becas.

- $\quad$ Proponer financiamiento para la consecución de títulos o certificados a diversos niveles, así como la realización de proyectos de investigación. 


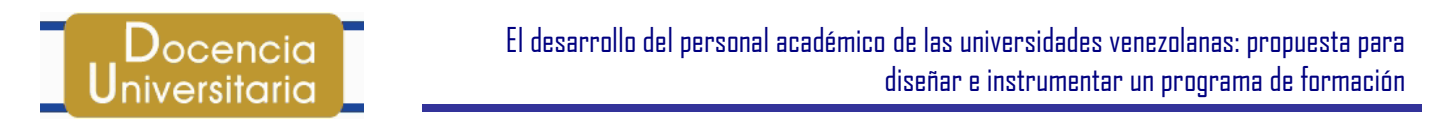

Aก̃̃ 3- N02- Dic. 2007

- Estimular a los profesores a elaborar portafolios individuales que den cuenta de su actividad académica, investigativa y de extensión universitaria.

La experiencia de las autoras permite destacar la importancia de la formulación e instrumentación de normativas adecuadas a cada institución para la regulación del desarrollo, formación y perfeccionamiento del personal académico. En efecto, las universidades venezolanas cuentan con estatutos que incluyen principios regulatorios de la carrera docente. Sin embargo, la mera estipulación reglamentaria no ha sido garantía de un avance ininterrumpido y sistemático de la carrera del profesor universitario.

En una normativa adecuada a la complejidad de los tiempos que corren, se tendría que establecer la obligación de todo profesor universitario de cumplir con un plan individual de carrera en la institución, en los plazos y condiciones ya indicadas. Asimismo, se deberían explicitar tanto los derechos y deberes que, al respecto, tienen los docentes universitarios, así como los compromisos institucionales en la materia. El espíritu general de dicha normativa será la creación de un clima institucional de compromiso hacia el programa que estimularía al profesor a un aprendizaje permanente. De este modo, se evitarían también desequilibrios e injusticias en la utilización de los recursos destinados a estos fines.

\section{Evaluación del programa}

La evaluación de un programa representa el proceso sistemático de recolección de información para la consecuente toma de decisiones sobre la continuidad o modificación del mismo. Permite conocer, verificar o incrementar el impacto de los productos en la clientela. Mejora los mecanismos de entrega para hacerlos más eficientes y a un menor costo. Determina lo que se "está haciendo" en relación con lo que "se piensa que se está haciendo”. Facilita el análisis que los administradores deben realizar sobre las metas fijadas y la forma de alcanzarlas. Y, finalmente, realiza comparaciones válidas entre programas para decidir los elementos que se deben mantener (Feasley, 1980).

Dada la complejidad que reviste la evaluación de un programa, se hace necesario recurrir a modelos que garanticen la objetividad del proceso evaluativo. Resulta de utilidad considerar los siguientes:

- $\quad$ La evaluación de la congruencia entre logros y objetivos, en la cual la calidad del programa se determina identificando la dimensión de la brecha existente entre lo planificado y lo obtenido. 


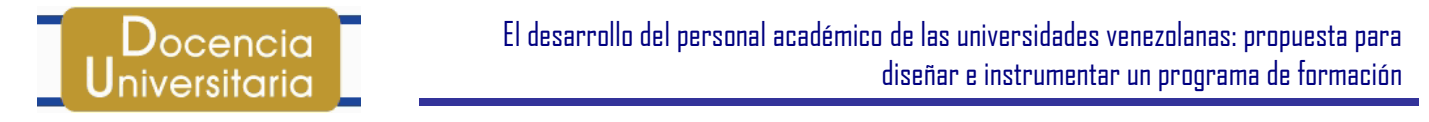

Аก̃̃ 3- N²- Dic. 2007

- La evaluación como juicio profesional, en el cual se consideran los insumos (los recursos materiales y humanos, instalaciones, personal de apoyo, presupuesto, entre otros), los procesos (la forma de entrega del programa) y los productos (las unidades de servicio en términos del número de personas asistidas).

- La evaluación de impacto, que toma como referencia la cantidad y calidad de los productos obtenidos.

La planificación evaluativa de un programa dependerá de los recursos con los que se dispongan, así como de la información que se espere obtener. Frecuentemente se quiere conocer "todo" sobre los productos elaborados. Sin embargo, se hace necesario establecer prioridades. Es recomendable identificar claramente los aspectos a evaluar, ya que mientras más focalizados se encuentren, más eficiente será la evaluación.

Asimismo, mientras menor sea el tiempo que se invierta en la conducción de la evaluación, más bajos serán los costos. Deberá considerarse también que mientras mayor sea el espectro que se desee cubrir en el proceso evaluativo, menor será la profundidad del análisis de cada aspecto y, por consiguiente, cuando se pretenda examinar a profundidad un elemento particular, probablemente no se obtenga información suficiente sobre otros rubros del programa

Tratando de ser consecuentes con lo explicado sobre las etapas del programa, debe ratificarse la importancia de que el proceso evaluativo se realice de forma continua y oportuna. En tal sentido, en la etapa de conceptualización se requiere la evaluación de las necesidades personales, institucionales y sociales para obtener información sobre el propósito y los objetivos que se persiguen, así como el tipo de programa que mejor responda a las necesidades planteadas. En la etapa de instrumentación resulta de vital importancia considerar el grado de avance del programa de acuerdo al logro progresivo de los objetivos. También deben tomarse en cuenta la fluidez y exactitud de los procesos, la cantidad y calidad de los recursos formados, y el mérito general del programa respecto a los efectos que produce. Esto proveerá a los responsables del mismo de una base objetiva para determinar la continuación, modificación o eliminación de algunos de sus elementos ${ }^{11}$.

Respecto a las estrategias para el diseño e instrumentación del proceso de evaluación de un programa de formación nos permitimos formular las siguientes recomendaciones:

\footnotetext{
${ }^{11}$ Feasley 1980
} 


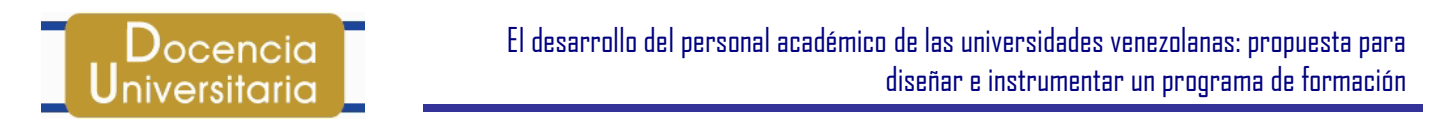

Аก̃̃ 3- N²- Dic. 2007

1. Recolectar información cualitativa y cuantitativa por medio del uso de cuestionarios, listas de cotejo, entrevistas, revisión documental, estudio de casos, análisis costo/beneficio, y análisis de efectividad y eficiencia. Esto permitirá conocer los resultados desde diferentes perspectivas.

2. Procesar la información obtenida a través de un cuidadoso sistema de recopilación de información, lo que permitirá el análisis objetivo de la data.

3. Organizar la información que será presentada en categorías, clasificándola por temas. Es necesaria la utilización de cuadros numéricos y gráficos lo suficientemente explícitos para una fácil interpretación. Será de gran ayuda quienes lean el informe la identificación de hallazgos, patrones y asociaciones, así como la formulación de sugerencias y recomendaciones para el equipo responsable del programa.

4. Presentar el reporte de resultados conforme a los objetivos de la evaluación, cotejando metas y logros, ya que esto permitirá organizar los datos y focalizar el análisis.

5. Considerar los recursos de los cuales se dispone (financieros y temporales) y la audiencia a la cual será destinada la información, pues aquello determinará las características del reporte evaluativo que se presentará.

Son dos las principales opciones que pueden tomarse respecto al (los) responsable (s) de la evaluación del programa: a) obtener asesoramiento externo a la institución para la evaluación; o b) determinar que la misma unidad que haya coordinado el programa de desarrollo lo evalúe. Sin embargo, los expertos en la materia no recomiendan esta segunda opción, ya que no garantiza la objetividad en el análisis de las debilidades y fortalezas del programa, pudiendo ser malinterpretados los datos. Por ello, es recomendable integrar al equipo de evaluadores por lo menos a un especialista externo, quien, gracias a su desvinculación con las etapas de diseño e instrumentación del programa, pueda evaluarlo sin estar contaminado con la operatividad del mismo.

\section{Consideraciones finales}

Las autoras desean ratificar la importancia de que los gerentes de las universidades combatan la rigidez organizativa e introduzcan los cambios que se requieren para el desarrollo del personal académico. Es el momento de reconsiderar la realidad educativa respecto a los procesos de desarrollo del profesor universitario que las instituciones de educación superior ofrecen. En tal sentido, es indispensable priorizar un programa de formación de profesores que responda a las exigencias de la sociedad, al marco de las 


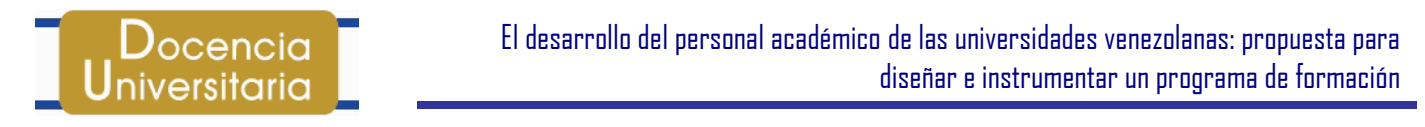

Аก̃̃ 3- N²- Dic. 2007

leyes y lineamientos de gestión establecidos periódicamente, y a lo requerido por las políticas y reglamentos internos de las instituciones.

Igualmente, debe considerarse necesario incrementar la eficiencia en el uso de los recursos financieros, pues la propia característica modélica de servicio social que prestan las universidades, implica la pulcritud en el manejo de los mismos, así como su adecuación y la pertinencia del costo-beneficio. Es importante dirigirse hacia la concreción de las metas propuestas por las instancias gubernamentales que, en el sentido del presente trabajo, implican:

- Implantar un sistema de desarrollo del personal académico que atienda las peculiaridades institucionales para incrementar la calidad.

- Diseñar un sistema nacional homologado de carrera que compense el esfuerzo.

- $\quad$ Elaborar planes integrales de formación que incorporen las dimensiones profesionales, institucionales y sociales.

Un programa de desarrollo del profesor universitario no estaría completo sin el aporte previo de un plan de carrera, el cual, desde el momento de la incorporación del profesor a la universidad, vaya reflejando sus intereses, potencialidades y aspiraciones. Entre ellas, debe incluirse la formación gerencial que permita al profesor ejercer labores administrativas para las cuales no siempre se encuentra debidamente preparado.

Finalmente, para la puesta en práctica de un programa de desarrollo de personal académico como el propuesto, resulta imprescindible que los deseos, necesidades y esfuerzos individuales e institucionales se enriquezcan con acciones de apoyo intra e interinstitucionales, pues ello potenciará un uso eficiente de los recursos y propiciará el intercambio entre organizaciones con objetivos comunes. La concreción de cada uno de estos aspectos, en opinión de las autoras, marcará una ruta verdadera hacia la mejora de la calidad de la enseñanza en las instituciones venezolanas de educación superior. 


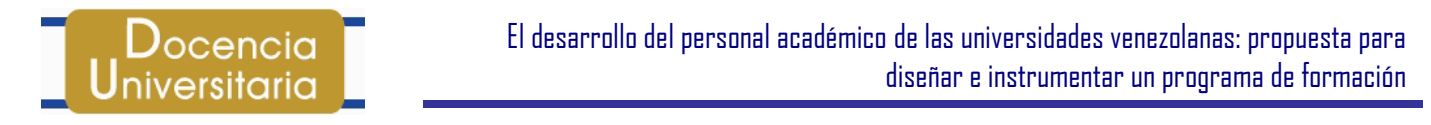

Аก๊̃ 3- №2- Dic. 2007

\section{Bibliografía}

ANDRADE, Francisco

2003 Plan de carrera: algunas ideas conceptuales para su implementación (consulta: 17 de julio del 2007).

(http://www.tress.com.mx/boletin/junio2003/plancarrera.htm)

AZÓCAR, L.; BENKO, A.; BERMÚDEZ, M.; LEAL, L. y RAMOS, Z.

1995 Orientaciones para la Implantación de un programa de desarrollo del personal académico de la UNA. UNA Documenta, No. 13 pp. 18-23.

CASTILLO, Santiago y CABRERIZO, Jesús

2005 Formación del Profesorado Universitario en Educación Superior. Vol. II. Madrid: McGraw Hill.

CERNA, Juan Carlos

2004 Aspectos básicos en la gestión del talento humano. En: (http://www.monografias.com/trabajos16/talento-humano/talentohumano.shtml?monosearch) (consulta: 17 de julio del 2007).

CHACÍN, Migdy y FIGUEROA, D.

1997 Plan de Desarrollo del Personal Docente y de Investigación. Caracas: UNESR.

CORTÉS, Aída y GONZÁLEZ, Raiza

2006 Programas de formación permanente del docente universitario en Venezuela. Revista OMNIA Año 12, No. 1. ISSN 1315-8856. pp 130-146.

CRAVENER, Patricia

2000 The Psychosocial System Checklist for Planning Faculty/Staff Development Programs. En: (www.cravener.net).

(consulta: 8 de julio del 2003).

DOLAN, Simón; VALLE, Ramón; JACKSON, Susan y SCHULLER, Randall

2007 La Gestión de los Recursos Humanos. 3ra.ed. Madrid: Mc Graw Hill.

FEASLEY, Charles

1980 Evaluación de Programas. En: American Association for Higher Education. Vol. 3. pp. 7-10. 


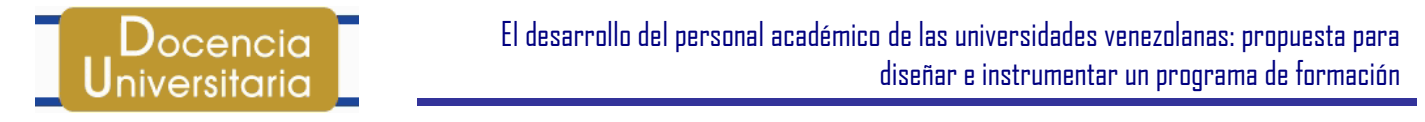

Аก̃̃ 3- N²- Dic. 2007

LAWLER, Patricia y KING, Kathleen

1999 Refocusing Faculty Development: the view from an adult education perspective. En: (http://www.edst.educ.ubc.ca/aerc/2000/lawlerp\&kingk-web.htm)

(consulta: 23 de junio del 2003).

MINISTERIO DE EDUCACIÓN, CULTURA Y DEPORTES.

2001 Políticas y estrategias para el desarrollo de la Educación Superior en Venezuela 2000-2006. CARACAS: Ministerio de Educación, Cultura y Deportes.

PEÑA, José

2007 Desarrollo profesional del docente universitario.

En: (http://www.oei.es/valores2/monografia03/reflexion03.htm) (consulta: 14 de julio del 2007).

SÁNCHEZ, José Antonio

El desarrollo profesional del docente universitario. Universidad Politécnica de Madrid.

En: (http://www.unam.mx/udual/cidu/22/DesarrolloProfesional) (consulta: 27 de julio del 2007).

UNELLEZ Universidad Experimental de los Llanos Ezequiel Zamora (s/f), Propuesta UNELLEZ. En: (http://www.unellez.edu.ve/default/mapa/prog.htm). (consulta: 19 de julio del 2003).

VILLARROEL, César

2007 La Calidad de la Educación Superior Latinoamericana. Caracas: CRESALCUNESCO.

\section{Para citar este documento, puede utilizar la siguiente referencia:}

CHACÍN, Migdy; DE KOLSTER, Maríangelina y RAMOS, Zobeida (2007). “ El desarrollo del personal académico de las universidades venezolanas: propuesta para diseñar e instrumentar un programa de formación” [artículo en línea]. Revista Digital de Investigación en Docencia Universitaria (RIDU) Año 3 - $N^{\circ} 2$-Diciembre 2007. [Fecha de consulta: dd/mm/aa]. <http://beta.upc.edu.pe/calidadeducativa/ridu/2007/ridu4 1ZR.pdf $>$ 


\section{Migdy N. Chacín.}

mchacin@cantv.net

Profesora de Biología y Química. PhD. Educación Superior. Post-doctorado Gerencia. Obras: Encuentro entre Líneas; Como Generar Líneas de Investigación; Cultura de la Evaluación en la Sociedad del Conocimiento; El currículo y la Formación de Investigadores; Investigación-Docencia. Fundadora y miembro línea de Investigación sobre la Función Docente-Universidad Simón Rodríguez. Programa de Promoción al Investigador PPI Venezuela, nivel I. y Programa de Estímulo Investigador -UNESR-.

\section{Maríangelina de Kolster}

lakolster@hotmail.com

Abogada. Profesora jubilada, miembro fundador de la Universidad Nacional Abierta. Magíster Scientiarum en Educación Superior de la Universidad Simón Rodríguez. Investigadora y articulista de educación a distancia, diseño instruccional y curricular y formación y capacitación de profesores universitarios. Funciones académicoadministrativas en la UNA y Gerente de Investigación y Desarrollo Educativo en el Centro de Formación de Petróleos de Venezuela (CEPET).

\section{Zobeida Ramos}

zobeidaramostovar@hotmail.com

Licenciada en Educación .Magister Scientiarum. Ecología Humana, PhD. Educación. Profesora Jubilada de la Universidad Nacional Abierta. Desempeño formación y el desarrollo de profesores universitarios. Coautora "Making Distance Education Work: understanding learning and learners at distance”. Investigadora y articulista en el área de desarrollo del personal académico-universitario. Miembro del Programa de Promoción al Investigador Venezuela 2006, nivel I. 